\title{
Expressions of multiple umami taste receptors in oral and gastrointestinal tissues, and umami taste synergism in chickens
}

\author{
Yuta Yoshida $^{\text {a }}$, Yuko Kawabata ${ }^{\text {a,b }}$, Fuminori Kawabata ${ }^{\text {c, }}$, \\ Shotaro Nishimura ${ }^{\mathrm{a}, \mathrm{b}}$, and Shoji Tabata ${ }^{\mathrm{a}, \mathrm{b}}$ \\ aLaboratory of Functional Anatomy, Department of Bioresource Sciences, Graduate \\ School of Bioresource and Bioenvironmental Sciences, Kyushu University, Fukuoka, \\ Japan \\ ${ }^{b}$ Faculty of Agriculture, Kyushu University, Fukuoka, Japan \\ 'Institute for Advanced Study, Kyushu University, Fukuoka, Japan
}

*Corresponding author: Fuminori Kawabata, Institute for Advanced Study, Kyushu University, 6-10-1 Hakozaki, Higashi-ku, Fukuoka 812-8581, Japan. Tel.: +81-92-642-2944. Fax: +81-92-642-2944

E-mail address: kawabata@agr.kyushu-u.ac.jp 


\section{ABSTRACT}

Umami taste is one of the five basic taste qualities, along with sweet, bitter, sour, and salty, and is elicited by some L-amino acids and their salts, including monopotassium L-glutamate (MPG). The unique characteristic of umami taste is that it is synergistically enhanced by 5'-ribonucleotides such as inosine 5'-monophosphate (IMP). Unlike the other four basic taste qualities, the presence of umami taste sense in avian species is not fully understood. In this study, we demonstrated the expression of multiple umami taste receptor candidates in oral and gastrointestinal tract tissues in chickens using RT-PCR analysis. We first showed the metabotropic glutamate receptors (mGluRs) expressed in these tissues. Furthermore, we examined the preference for umami taste in chickens, focusing on the synergistic effect of umami taste as determined by the two-feed choice test. We concluded that chickens preferred feed containing both added MPG and added IMP over feeds containing either added MPG or added IMP alone and over the control feed. These results suggest that the umami taste sense and synergism are conserved in chickens. 
Key words: umami, mGluR1, mGluR4, T1R1, T1R3, chicken

\section{Introduction}

In chickens, the taste sense is one of the most important senses for acquiring and choosing feeds, as are the smell and visual senses [1]. It is important to clarify the mechanism of taste sense in chickens in order to improve the efficiency of poultry feeding through the feeding of preferable feed and to elucidate the evolution of the taste sense from birds to mammals.

Umami taste is one of the five basic taste qualities, and is discriminated from the other four basic taste qualities (sweet, bitter, sour, and salty) in humans $[2,3,4]$. L-glutamate and its salts, including monosodium L-glutamate and monopotassium L-glutamate (MPG), are used as seasonings and are known to be umami tastants for humans. Interestingly, the umami taste is synergistically potentiated by 5'-ribonucleotides, such as inosine 5'-monophosphate (IMP), which is contained in some foods such as dried bonito $[5,6]$. The umami taste receptor candidates in mammals are the G-protein-coupled receptors, the heterodimer of taste receptor type 1 (T1R1) and 
taste receptor type 3 (T1R3) and brain-expressed and taste-expressed type 1 and type 4 metabotropic glutamate receptors (brain-mGluR1, brain-mGluR4, taste-mGluR1, and taste-mGluR4, respectively) $[7,8,9,10]$. The umami taste synergism can be explained by stabilization of the conformation of the extracellular domain of T1R1 by its allosteric modulator, IMP [11].

Recently, many reports related to the taste sense in chickens have been published. Behrens et al. reported the activities of three chicken bitter taste receptors, the type 2 taste receptors (T2R1, T2R2, and T2R7), using a cell-based assay [12]. Hirose et al. found that the activities of T2R1 were compatible with behavioral sensitivities to bitterness in chickens [13]. Sawamura et al. revealed that a chicken fatty acid receptor candidate, GPR120 (G-protein-coupled receptor 120), cloned from chicken oral tissue, responded to fatty acids [14]. With respect to the sense of umami taste in chickens, one of the umami taste receptors, the T1R1/T1R3 heterodimer, was expressed in the oral and gastrointestinal tract tissues in chickens [15] and was activated by L-alanine and L-serine [16]. In another study, we revealed that primary culture cells from isolated taste buds from the chicken palate responded to a mixture of MPG and IMP [17]. Here, we 
speculated that, if the umami taste sense and its unique characteristic, umami taste synergism, were conserved in chickens, feed intakes would be synergistically enhanced by umami taste enhancers such as IMP. In this study, we analyzed the expression of multiple umami taste receptor gene candidates by RT-PCR and tested the feed preference for umami tastants focusing on umami taste synergism, which is specific to this taste, using the two-feed choice test in chickens.

\section{Materials and methods}

\subsection{Chemicals}

MPG and IMP were purchased from Sigma-Aldrich (Tokyo). These compounds were stored at room temperature.

\subsection{Animals}

Rhode Island Red strain 3-day-old chicks were used for the RT-PCR, and 0 -week-old chicks were used for the two-feed choice test. These studies were carried out according to the Guide for Animal Experiments issued by Kyushu University, the 
Law Concerning the Human Care and Control of Animals (Law No. 105; October 1, 1973), and the Japanese Government Notification on the Feeding and Safekeeping of Animals (Notification No. 6; March 27, 1980).

\subsection{RT-PCR analysis}

Total RNA was isolated from the brain, oral tissues (palate beak, tongue tip, and floor beak), and gastrointestinal tissues (ingluvies, stomach, gizzard, duodenum, jejunum, ileum, and colon), using ISOGEN II (Nippon Gene Co., Ltd., Tokyo) according to the manufacturer's instructions. The first-strand cDNA was synthesized by reverse transcription, with the application of $1.0 \mu \mathrm{g}$ total RNA with or without reverse transcriptase using the PrimeScript RT reagent kit with gDNA Eraser (TaKaRa Bio Inc., Otsu, Japan) following the manufacturer's protocol. Primers were designed with the aid of the nucleotide database of The National Center for Biotechnology Information and are shown in Table 1. Although the primer set of brain-mGluR1 has the ability to amplify two splice variants of brain-mGluR1 (Accession no. XM_004935585.1 and XM_419652.3), we thought it reasonable to elucidate the whole expression patterns of 
brain-mGluR1 without discriminating among these variants in this study. The PCR mixture had a total volume of $10 \mu 1$ and consisted of $5.85 \mu 1$ distilled water, $1.0 \mu 110 \times$ Ex Taq buffer (TaKaRa Bio Inc.), $0.8 \mu \mathrm{l}$ dNTP mixture (2.5 mM) (TaKaRa Bio Inc.), $0.4 \mu 1$ primer forward $(10 \mu \mathrm{M}), 0.4 \mu 1$ primer reverse $(10 \mu \mathrm{M}), 1.5 \mu \mathrm{l}$ cDNA $(100 \mathrm{ng} / \mu \mathrm{l})$,

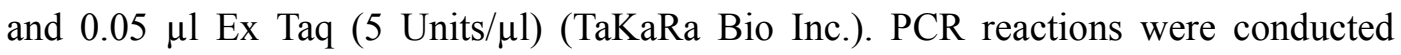
under the following conditions: $38-40$ cycles of $98^{\circ} \mathrm{C}$ for $10 \mathrm{sec}, 60^{\circ} \mathrm{C}$ for $30 \mathrm{sec}$, and $72^{\circ} \mathrm{C}$ for $1 \mathrm{~min} / 1 \mathrm{~kb}(19-48 \mathrm{sec})$. Five $\mu \mathrm{l}$ PCR products were electrophoresed on a $2.0 \%$ agarose gel.

\subsection{Two-feed choice test}

This experiment was conducted using a box brooder (Showa Furanki, Saitama, Japan) to keep the temperature at $30^{\circ} \mathrm{C}$. The box brooder was partitioned using woven metal wire so that the chicks could see other chicks through the wire. The chicks could freely drink tap water during the entire experimental period. As the experimental feed, PL1 (Oriental Yeast Co., Tokyo) was used. The feed is a normal and suitable feed for fulfilling the nutritional requirements of chicks. First, the umami feed (PL1 with the 
addition of either MPG or IMP or both) was presented to the chicks for $24 \mathrm{~h}$, and then the umami feed and the control feed (PL1) were presented simultaneously to the chicks for $7 \mathrm{~h}$. This training period was conducted to help them become accustomed to the experimental condition. After that, the chicks fasted for $17 \mathrm{~h}$. The umami feed and the control feed were then presented simultaneously to the chicks again for $7 \mathrm{~h}$, and the feed intake for this $7 \mathrm{~h}$ period was measured. This test was carried out for each concentration of umami substances, using 8-12 chicks, and for each of 5 conditions: 1) $0.05 \%$ MPG, 0.005\% IMP, and 99.945\% PL1; 2) 0.5\% MPG, 0.05\% IMP, and 99.45\% PL1; 3) 5\% MPG, 0.5\% IMP, and 94.5\% PL1; 4) 0.55\% MPG and 99.45\% PL1; and 5) 0.55\% IMP and $99.45 \%$ PL1.

\subsection{Statistical Analysis}

The data are expressed as the means \pm SEM. Statistical analyses were done by paired $t$-test. Differences with $p$-values $<0.05$ were considered significant.

\section{Results}




\subsection{RT-PCR analysis}

RT-PCR analysis showed that the expression of multiple umami taste receptor candidates, namely taste-mGluR1, brain-mGluR1, taste-mGluR4, brain-mGluR4, and T1R1/T1R3, was observed in the brain, palate beak, floor beak, ingluvies, stomach, gizzard, duodenum, jejunum, ileum, and colon in chickens, but not in the tongue tip, where almost no taste buds are distributed (Fig. 1A) [18]. We confirmed that no band was observed in the negative control reactions without reverse transcriptase (Fig. 1B).

\subsection{The two-feed choice test}

In the two-feed choice test, the intakes of the feed with the addition of both $0.5 \%$ MPG and $0.05 \%$ IMP were significantly larger than those of the control feed (Fig. 2).

On the other hand, the intakes of the feed with the addition of either $0.55 \%$ MPG or 0.55\% IMP showed no significant differences with those of the control feed (Fig. 2). The addition of both MPG and IMP at higher concentrations (5\% MPG and 0.5\% IMP) and lower concentrations ( $0.05 \%$ MPG and $0.005 \%$ IMP) had no significant effect on the feed intakes compared with those of the control feed (Fig. 2). 


\section{Discussion}

In the present study, the RT-PCR analysis showed that multiple umami taste receptor candidates, namely taste-mGluR1, brain-mGluR1, taste-mGluR4, brain-mGluR4, and T1R1/T1R3, were expressed in the oral and gastrointestinal tissues except for the tongue tip in chickens (Fig. 1). We first revealed that mGluRs is expressed in these tissues. We previously studied the distribution of taste buds in chicken oral tissues and reported that the taste buds were observed mainly in the palate beak, the floor beak, and the root of the tongue, with a few in the tongue tip [18]. Therefore, because no umami taste receptors were observed in the tongue tip in chickens, the umami taste receptors found in the oral tissues may be specifically expressed in the taste buds. These data suggest the possibility that chickens sense umami taste using multiple umami taste receptors in the oral tissues, as mammals do [19].

In mammals, the gut-expressed T1R1/T1R3 stimulates cholecystokinin secretion [20], and the gut-expressed sweet taste receptor, the T1R2/T1R3 heterodimer, and the 
taste $\mathrm{G}$ protein, gustducin, regulate glucagon-like peptide-1 secretion [21,22,23]. The activation of T1R1/T1R3 expressed in the colon elicits the peristaltic reflex and pellet propulsion in mammals [24]. In regard to the gastrointestinal mGluRs, Akiba et al. reported that mGluR1 and mGluR4 expressed in the duodenum regulate the duodenal mucosal defense to acidic stimulus [25]. Thus, we speculate that gut-expressed umami taste receptors in chickens may also play roles in regulating the secretion of the gut hormones, maintaining the gut motility, and regulating the mucosal defense. Since the physiological roles of the umami taste receptors expressed in the gastrointestinal tract in chickens have not been elucidated, further studies are needed. Obtaining information regarding these roles is very important because controlling the appetite and gut health of chickens is directly related to the efficiency of poultry farming. Furthermore, the elucidation of the functions of the umami taste receptors in the ingluvies and gizzard, which are avian-specific organs, will help to elucidate avian physiology.

In the two-feed choice test, it was suggested that chickens preferred the feed with the addition of both MPG and IMP over the feed with the addition of either MPG or IMP (Fig. 2). These results can be explained by umami taste synergism, the 
enhancement of umami taste intensity by IMP. These results suggest that chickens have umami taste sense and synergism, and umami taste synergism is conserved from birds to mammals.

However, in spite of the addition of both of MPG and IMP, the addition of 5\% MPG and 0.5\% IMP (higher concentrations), and 0.05\% MPG and 0.005\% IMP (lower concentrations) had no significant effect on the feed intakes compared with the control feed (Fig. 2). Previous studies have shown that the supplementation of $1 \%$ L-glutamate in feed without adequate amino acid balance had an even more negative impact on the appetites of broiler chickens [26] and 5\% $\mathrm{NaCl}$ in feed elicited aversion in chickens [27]. Therefore, in the case of the addition of 5\% MPG and 0.5\% IMP, it is speculated that the excess L-glutamate and salts contained in the MPG and IMP were not preferable to chickens. On the contrary, in the case of the addition of $0.05 \%$ MPG and $0.005 \%$ IMP, it is thought that the concentration did not reach the umami taste threshold of chickens. Therefore, these results suggest that there is an appropriate range of the concentrations of MPG and IMP that is preferred in chickens.

In conclusion, we found that multiple umami taste receptors are expressed in the 
oral and gastrointestinal tract tissues in chickens and that supplementation of feed with an umami tastant and its enhancer causes a synergistic effect on feed intakes in chickens.

These results support the presence of umami taste sense and synergism in chickens, provide important information regarding the evolution of umami taste sensing, and may help improve the feed intakes of chickens in poultry farming.

\section{Acknowledgments}

We appreciate the technical assistance of The Research Support Center, Research Center for Human Disease Modeling, Kyushu University Graduate School of Medical Sciences.

This study was supported in part by a grant to F.K. from the Funds for the Development

of Human Resources in Science and Technology, the Japan Science and Technology Agency; a grant to F.K. from JSPS KAKENHI (\# 26850207); and a grant to F.K. from Kyushu University Interdisciplinary Programs in Education and Projects in Research Development (\#26703).

\section{Conflict of interest}

None. 


\section{References}

[1] E. Roura, M.W. Baldwin, K.C. Klasing, The avian taste system: Potential implications in poultry nutrition, Animal Feed Science and Technology 180 (2013) 1-9.

[2] G. Hellekant, V. Danilova, Y. Ninomiya, Primate sense of taste: behavioral and single chorda tympani and glossopharyngeal nerve fiber recordings in the rhesus monkey, Macaca mulatta, J. Neurophysiol. 77 (1997) 978-993.

[3] Y. Ninomiya, M. Funakoshi, Peripheral neural basis for behavioural discrimination between glutamate and the four basic taste substances in mice, Comp. Biochem. Physiol. A. Comp. Physiol. 92 (1989) 371-376.

[4] Y. Ninomiya, M. Funakoshi, Behavioral discrimination between glutamate and the four basic taste substances in mice, Comp. Biochem. Physiol. A. Comp. Physiol. 92 (1989) 365-370.

[5] S. Yamaguchi, The synergistic taste effect of monosodium glutamate and disodium 5' -inosinate, J. Food Sci. 32 (1967) 473-478.

[6] S. Yamaguchi, Basic properties of umami and effects on humans, Physiol. Behav. 49 (1991) 833-841.

[7] N. Chaudhari, H. Yang, C. Lamp, E. Delay, C. Cartford, T. Than, S. Roper, The taste of monosodium glutamate: membrane receptors in taste buds, J. Neurosci. 16 (1996) 3817-3826.

[8] N. Chaudhari, A.M. Landin, S.D. Roper, A metabotropic glutamate receptor variant functions as a taste receptor, Nat. Neurosci. 3 (2000) 113-119.

[9] G. Nelson, J. Chandrashekar, M.A. Hoon, L. Feng, G. Zhao, N.J. Ryba, C.S. Zuker, An amino-acid taste receptor, Nature 416 (2002) 199-202.

[10] X. Li, L. Staszewski, H. Xu, K. Durick, M. Zoller, E. Adler, Human receptors for sweet and umami taste, Proc. Natl. Acad. Sci. U S A 99 (2002) 4692-4696.

[11] F. Zhang, B. Klebansky, R.M. Fine, H. Xu, A. Pronin, H. Liu, C. 
Tachdjian, X. Li, Molecular mechanism for the umami taste synergism, Proc. Natl. Acad. Sci. U S A 105 (2008) 20930-20934.

[12] M. Behrens, S.I. Korsching, W. Meyerhof, Tuning properties of avian and frog bitter taste receptors dynamically fit gene repertoire sizes, Molecular Biology and Evolution 31 (2014) 3216-3227.

[13] N. Hirose, Y. Kawabata, F. Kawabata, S. Nishimura, S. Tabata, Bitter taste receptor $\mathrm{T} 2 \mathrm{R} 1$ activities were compatible with behavioral sensitivity to bitterness in chickens, Biochem. Biophys. Res. Commun. 460 (2015) 464-468.

[14] R. Sawamura, Y. Kawabata, F. Kawabata, S. Nishimura, S. Tabata, The role of G-protein-coupled receptor 120 in fatty acids sensing in chicken oral tissues, Biochem. Biophys. Res. Commun. 458 (2015) 387-391.

[15] S.L. Cheled-Shoval, S. Druyan, Z. Uni, Bitter, sweet and umami taste receptors and downstream signaling effectors: Expression in embryonic and growing chicken gastrointestinal tract, Poult. Sci. 94 (2015) 1928-1941.

[16] M.W. Baldwin, Y. Toda, T. Nakagita, M.J. O'Connell, K.C. Klasing, T. Misaka, S.V. Edwards, S.D. Liberles, Evolution of sweet taste perception in hummingbirds by transformation of the ancestral umami receptor, Science 345 (2014) 929-933.

[17] K. Kudo, F. Kawabata, T. Nomura, A. Aridome, S. Nishimura, S. Tabata, Isolation of chicken taste buds for real-time Ca2+ imaging, Anim. Sci. J. 85 (2014) 904-909.

[18] K. Kudo, S. Nishimura, S. Tabata, Distribution of taste buds in layer-type chickens: Scanning electron microscopic observations, Animal Science Journal 79 (2008) 680-685.

[19] K. Yasumatsu, N. Horio, Y. Murata, S. Shirosaki, T. Ohkuri, R. Yoshida, Y. Ninomiya, Multiple receptors underlie glutamate taste responses in mice, Am. J. Clin. Nutr. 90 (2009) 747S-752S.

[20] K. Daly, M. Al-Rammahi, A. Moran, M. Marcello, Y. Ninomiya, S.P. Shirazi-Beechey, Sensing of amino acids by the gut-expressed taste receptor T1R1-T1R3 stimulates CCK secretion, Am. J. Physiol.

Gastrointest. Liver Physiol. 304 (2013) G271-282. 
[21] H.J. Jang, Z. Kokrashvili, M.J. Theodorakis, O.D. Carlson, B.J. Kim, J. Zhou, H.H. Kim, X. Xu, S.L. Chan, M. Juhaszova, M. Bernier, B. Mosinger, R.F. Margolskee, J.M. Egan, Gut-expressed gustducin and taste receptors regulate secretion of glucagon-like peptide-1, Proc. Natl. Acad. Sci. U S A 104 (2007) 15069-15074.

[22] R.E. Steinert, A.C. Gerspach, H. Gutmann, L. Asarian, J. Drewe, C. Beglinger, The functional involvement of gut-expressed sweet taste receptors in glucose-stimulated secretion of glucagon-like peptide-1 (GLP-1) and peptide YY (PYY), Clin. Nutr. 30 (2011) 524-532.

[23] Z. Kokrashvili, B. Mosinger, R.F. Margolskee, Taste signaling elements expressed in gut enteroendocrine cells regulate nutrient-responsive secretion of gut hormones, Am. J. Clin. Nutr. 90 (2009) 822S-825S.

[24] D.M. Kendig, N.R. Hurst, Z.L. Bradley, S. Mahavadi, J.F. Kuemmerle, V. Lyall, J. DeSimone, K.S. Murthy, J.R. Grider, Activation of the umami taste receptor (T1R1/T1R3) initiates the peristaltic reflex and pellet propulsion in the distal colon, Am. J. Physiol. Gastrointest. Liver Physiol. 307 (2014) G1100-1107.

[25] Y. Akiba, C. Watanabe, M. Mizumori, J.D. Kaunitz, Luminal L-glutamate enhances duodenal mucosal defense mechanisms via multiple glutamate receptors in rats, Am. J. Physiol. Gastrointest. Liver Physiol. 297 (2009) G781-791.

[26] B.J. Kerr, M.T. Kidd, Amino acid supplementation of low-protein broiler diets: 1. Glutamic acid and indispensable amino acid supplementation, J. Appl. Poultry Res. 8 (1999) 298-309.

[27] J.M. Balog, R.I. Millar, Influence of the sense of taste on broiler chick feed consumption, Poult. Sci. 68 (1989) 1519-1526.

\section{Figure legends}


Fig. 1. Expression of multiple umami taste receptor gene candidates, namely taste-mGluR1, brain-mGluR1, taste-mGluR4, brain-mGluR4, and T1R1/T1R3, and the house-keeping gene, $\beta$-actin, in chicken oral and gastrointestinal tissues. (A) The expression of taste-mGluR1, brain-mGluR1, taste-mGluR4, brain-mGluR4, and T1R1/T1R3 was observed in the brain, the oral tissues (palate beak, floor beak), and the gastrointestinal tissues (ingluvies, stomach, gizzard, duodenum, jejunum, ileum, colon), but not in the tongue tip, which does not contain taste buds, by RT-PCR with reverse transcriptase $(\mathrm{RT}+)$. (B) There were no bands in the negative control reactions without reverse transcriptase $(\mathrm{RT}-)$.

Fig. 2. The two-feed choice test demonstrating feed intakes per body weight of the umami feed and control feed for $7 \mathrm{~h}$ in chickens. The intake of the feed with the addition of $0.5 \%$ MPG and $0.05 \%$ IMP was significantly larger than that of the control feed. These tests were performed separately for each concentration, and 8-12 chicks were used for each test. Values are the means \pm SEM $(n=8-12) .{ }^{*} p<0.05$ by paired $t$-test. 
Table 1. Primers used for the RT-PCR

\begin{tabular}{lllll}
\hline Target gene & Accession no. & Primer forward & Primer reverse & Product size (bp) \\
\hline Taste-mGluR1 & XM_004935586.1 & CATCATAGCCAAGCCCGAGA & TCTGATGAAGTCCTGGGTGC & 219 \\
Brain-mGluR1 & XM_004935585.1 & GGAGACACGTCTCTTCCTGC & TAGGTCATGTCTGGGGGTGT & 456 \\
& XM_419652.3 & & & 309 \\
Taste-mGluR4 & XM_004935045.1 & CTCTCCCGTCTCCAACCAAA & GATCATGCTCTACACAGTCATCG & 309 \\
Brain-mGluR4 & XM_003642745.2 & GACAACAACCGCCGAAACAT & CACCATCCACTGGGTCCATC & 271 \\
T1R1 & XM_425734.4 & CAGCTACGAAGCCTCTCTGG & GTAGGAGCTGCCAGGGATAG & 794 \\
T1R3 & XM_425740.3 & TGTTACGACCGCAGTGAGAG & GGGAACTCTGTGAGCAGGAC & 335 \\
B-actin & NM_205518.1 & ACACGGTATTGTCACCAACT & TAACACCATCACCAGAGTCC & 263 \\
\hline
\end{tabular}


(A) $\mathrm{RT}+$

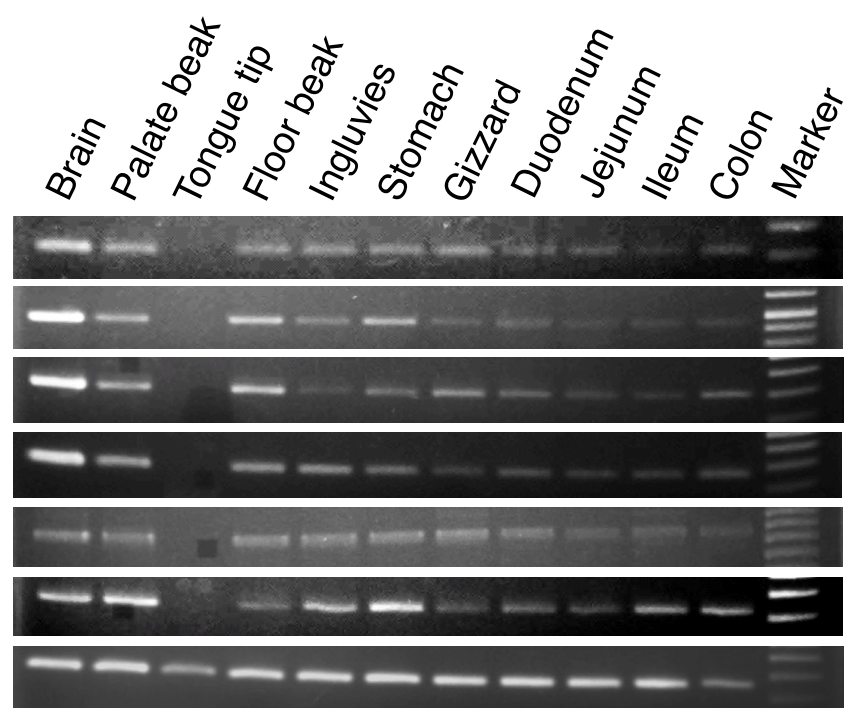

(B) $\mathrm{RT}-$

Taste-mGluR1

Brain-mGluR1

Taste-mGluR4

Brain-mGluR4

T1R1

$T 1 R 3$

$\beta$-actin

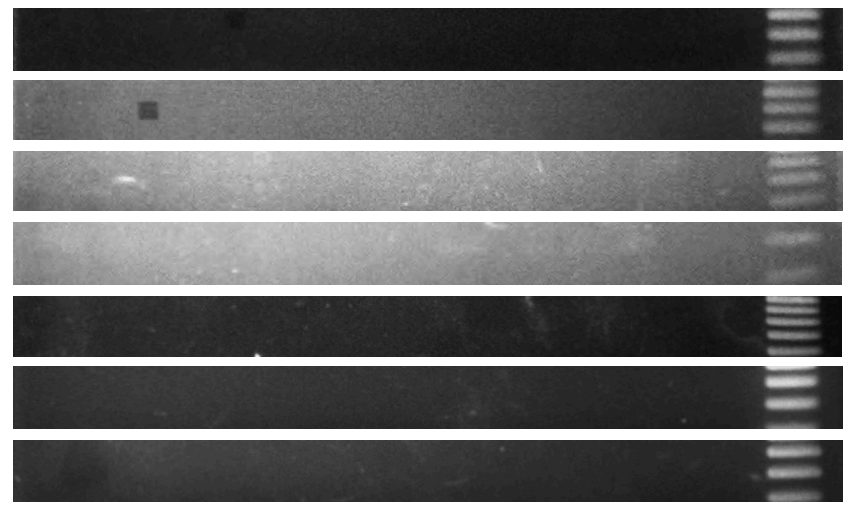

Figure 1

Yoshida et al. 


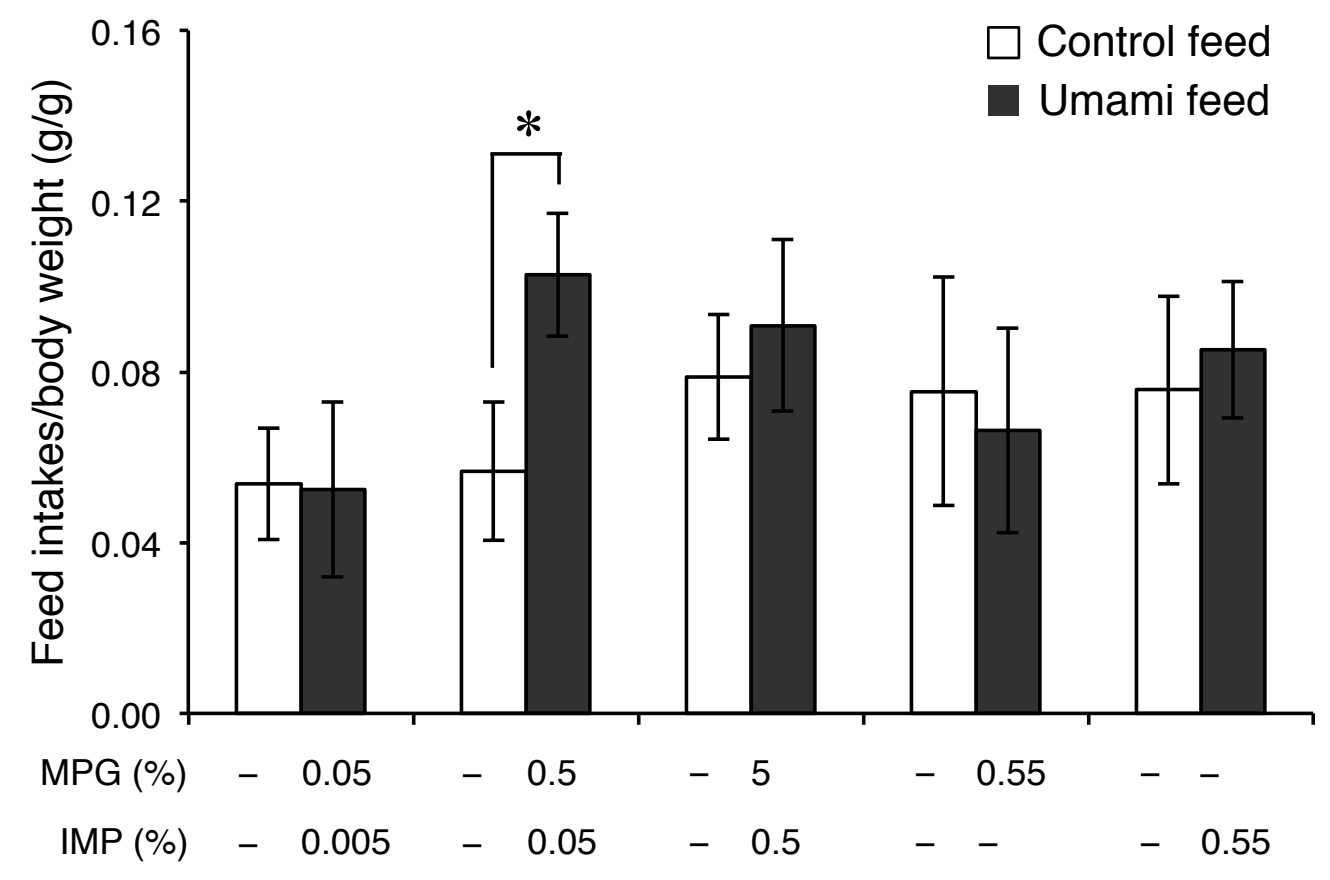

Figure 2

Yoshida et al. 